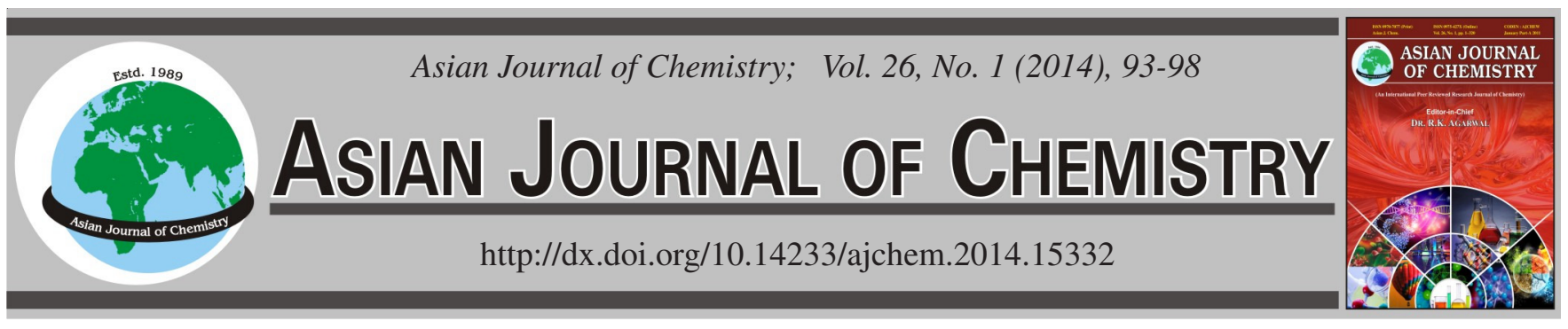

\title{
Synthesis, Spectral and Enzyme Inhibition Studies on $N$-Aralkyl/ Aryl Sulfonated Derivatives of Commercially Available Paroxetine
}

\author{
Asia Siddiqa ${ }^{1}$, Aziz-ur-Rehman ${ }^{1, *}$, Muhammad Athar Abbasi ${ }^{1}$, Shahid Rasool ${ }^{1}$, \\ Ghulam Hussain ${ }^{1}$, Khalid Mohammed Khan ${ }^{2}$, Muhammad Ashraf ${ }^{3}$ and Rumana Nasar ${ }^{4}$
}

\begin{abstract}
${ }^{1}$ Department of Chemistry, Government College University, Lahore, Pakistan
${ }^{2}$ HEJ Research Institute of Chemistry, International Center for Chemical and Biological Sciences, University of Karachi, Karachi, Pakistan ${ }^{3}$ Department of Biochemistry \& Biotechnology, The Islamia University of Bahawalpur, Bahawalpur, Pakistan

${ }^{4}$ Department of Pharmacy, The Islamia University of Bahawalpur, Bahawalpur, Pakistan
\end{abstract}

*Corresponding author: Tel: +92 42 111000010, Ext. 450; E-mail: azizryk@yahoo.com

\begin{abstract}
In the current work, a facile and environmentally benign series of $N$-aralkyl/aryl sulfonyl paroxetine 3a-n was synthesized and screened against lipoxygenase enzyme. These were geared up by the coupling of paroxetine (1) with different aralkyl/aryl sulfonyl chlorides, 2a-n, under dynamic $\mathrm{pH}$ control in aqueous basic media to form various $N$-substituted paroxetine (3a-l). The synthesized compounds were characterized by spectral data like IR, ${ }^{1} \mathrm{H}$ NMR, ${ }^{13} \mathrm{C}$ NMR and EI-MS. The bioactivity of all the synthesized compounds was evaluated against lipoxygenase (LOX) enzyme. Only six synthesized compounds showed moderate activity but all others remained inactive against lipoxygenase enzyme as it was evident from their $\mathrm{IC}_{50}$ values, relative to the standard used.
\end{abstract}

Keywords: Aralkyl/aryl sulfonyl chlorides, Paroxetine, Enzyme inhibition activity, ${ }^{1} \mathrm{H}$ NMR, ${ }^{13} \mathrm{C}$ NMR, IR, EI-MS.

\section{INTRODUCTION}

Paroxetine has potential inhibition and selectivity for 5-hydroxytryptamine (5-HTT, serotonin) uptake, having lessened tendency to execute the fallouts ordinarily associated with a tricyclic antidepressant ${ }^{1-3}$. The metabolic pathway of paroxetine was studied using $\left[{ }^{14} \mathrm{C}\right]$-labeled paroxetine and revealed (3S,4R)-trans-4-(4-fluorophenyl)-3-hydroxymethylpiperidine as one of the metabolites, which seems to be an attractive intermediate for the preparation of paroxetine ${ }^{2}$. Paroxetine is used during the treatment of depression, obsessive compulsive disorder and panic disorder. Paroxetine is an enantiomerically pure (-)-trans-3,4-disubstituted piperidine and because of its biological grandness, various enantiocontrolled syntheses have been divulged ${ }^{3}$.

Sulfonamides are pharmacologically significant compounds and largely used as carbonic anhydrase inhibitors; anticancer, antiinflammatory, antiviral agents; antimicrobial drugs, insulinreleasing sulfonamides; saluretics, antihydrod agents, antitumor drugs etc. These are the widely used as antibacterial agents because of their low cost, less toxicity and extraordinary activity $^{4-8}$.

Lipoxygenase (LOX, EC 1.13.11.12) belonging to a class of non-haem iron possessing dioxygenases, are widely occupied in animals and plants. These are implied in arachidonic acid metabolism and generation of various biologically active lipids which own a primary role in inflammation. These are also involved in thrombosis and tumor angiogenesis, organization of newfangled capillary vessels from pre-existing ones; support a large number of physiological processes and necessitate in the development of several pathological conditions such as arthritis and cancer. Lipoxygenases are, therefore, likely objectives for rational medicine design and also for the discovery of mechanism-based inhibitors for the treatment of a variety of disorders such as bronchial asthma, inflammation, cancer and autoimmune diseases ${ }^{9-11}$.

In this paper we have reported the synthesis of $N$-aralkyl/ aryl sulfonyl paroxetine and screened against lipoxygenase enzyme to find out their activity. In continuation of our research work $^{12,13}$ on sulfonamide synthesis and their derivatives, we have extended here our research work of synthesis with an intention to search new contenders of drug having significant activity and could be supportive in controlling many degenerative diseases.

\section{EXPERIMENTAL}

General: Aryl/aralkyl sulfonyl chlorides were purchased from Merck and Alfa Aeser through local suppliers and were 
used without further purification. Paroxetine was obtained from a local pharmaceutical industry and was used without any further purification and also its structure was corroborated via ${ }^{1} \mathrm{H}$ and ${ }^{13} \mathrm{C}$ NMR. All the solvents used, were of analytical grade. Purity of the synthesized compounds was checked by thin layer chromatography (TLC) with solvent systems using ethyl acetate and $n$-hexane. TLC plates were purchased from local supplier. TLC plates were visualized under UV at 254 $\mathrm{nm}$ and also by spraying with ceric sulfate solution. The IR spectra were recorded in KBr pellet method on a Jasco-320-A spectrophotometer (wave number in $\mathrm{cm}^{-1}$ ). Melting points of all the synthesized compounds were recorded on a GriffinGeorge melting point apparatus by open capillary tube and were uncorrected. ${ }^{1} \mathrm{H}$ and ${ }^{13} \mathrm{C}$ NMR spectra were recorded in $\mathrm{CDCl}_{3}$ on a Bruker spectrometers operating at 300/400 and $100 \mathrm{MHz}$, respectively. The chemical shift values are reported in ppm $(\delta)$ units taking TMS as reference and the coupling constant $(J)$ is in Hz. Mass spectra (EI-MS) were recorded on a JMS-HX-110 spectrometer.

General procedure for the synthesis of aryl/aralkyl sulfonyl paroxitine derivatives in aqueous media (3a-n): Paroxetine $(0.001 \mathrm{~mol} ; \mathbf{1})$ was dispersed in $100 \mathrm{~mL} \mathrm{RB}$ flask containing $30 \mathrm{~mL}$ water. The $\mathrm{pH}$ of solution was made 9-10 by $10 \%$ aqueous solution of $\mathrm{Na}_{2} \mathrm{CO}_{3}$ and maintained during the whole reaction. Aryl/aralkyl sulfonyl chlorides ( $0.001 \mathrm{~mol}$; 2a-n) were added in the basic solution in small portions at $\mathrm{pH}$ of 9-10. The reaction contents were kept on continuous stirring for $3 \mathrm{~h}$. The reaction progress was monitored through TLC using $n$-hexane and ethyl acetate as solvent system (70:30). After reaction completion, dilute $\mathrm{HCl}$ was poured slowly to adjust the $\mathrm{pH}$ of 2-3. The solid precipitates were filtered, washed with distilled water and dried to yield the corresponding compounds, 3a-n. Recrystallization was also processed for further purification from methanol.

Lipoxygenase assay: The lipoxygenase activity was carry out according to the reported method ${ }^{14}$ with slight modification. A total vol. of $200 \mu \mathrm{L}$ assay mixture containing $150 \mu \mathrm{L}$ buffer $\left(\mathrm{Na}_{3} \mathrm{PO}_{4}, 100 \mathrm{mM}, \mathrm{pH} 8.0\right), 10 \mu \mathrm{L}$ test compound and $15 \mu \mathrm{L}$ purified lipoxygenase enzyme (Sigma, USA). The contents were mixed, pre-read at $234 \mathrm{~nm}$ and pre-incubated for $10 \mathrm{~min}$ at $25^{\circ} \mathrm{C}$. The addition of $25 \mu \mathrm{L}$ substrate solution inducted the reaction. The change in absorbance was observed after a time of about $6 \mathrm{~min}$ at $234 \mathrm{~nm}$. Synergy HT (BioTek, USA) 96well plate reader was used in all experiments. All reactions were performed in triplicates. Baicalein $\left(0.5 \mathrm{mM}\right.$ well $\left.{ }^{-1}\right)$ was used as a positive control. The percentage inhibition and $\mathrm{IC}_{50}$ values were calculated as,

$$
\text { Inhibition }(\%)=\frac{\text { Control }- \text { Test }}{\text { Control }} \times 100
$$

$\mathrm{IC}_{50}$ values (concentration of compound at which $50 \%$ enzyme is inhibited) of compound were computed by EZ-fit enzyme kinetics software (Perella Scientific Inc. Amherst, USA). IC $_{50}$ values were calculated (as mean of three independent experiments) from the graph by dilution of compounds to different concentrations.

Statistical analysis: All the measurements were done in triplicate and statistical analysis was performed by Microsoft Excel 2010. Results are presented as mean \pm sem.

\section{Spectral characterization of the synthesized compounds}

$\boldsymbol{N}$-(Benzenesulfonyl)paroxetine (3a): White amorphous solid; Yield: $85 \%$; m.p.: $205^{\circ} \mathrm{C}$; m.f.: $\mathrm{C}_{25} \mathrm{H}_{24} \mathrm{NO}_{5}$ SF; molecular mass: $469 ;{ }^{1} \mathrm{H} \mathrm{NMR}\left(\mathrm{CDCl}_{3}, 300 \mathrm{MH}_{\mathrm{Z}}\right): \delta(\mathrm{ppm}): 7.78(\mathrm{dd}$, $J=6.9,1.5 \mathrm{~Hz}, 2 \mathrm{H}, \mathrm{H}-2$ "' $^{\prime}$ and H-6"'), 7.63-7.61 (m, 1H, H4"'), 7.58 (dd, $J=6.9,1.5 \mathrm{~Hz}, 2 \mathrm{H}, \mathrm{H}-3$ "' and H-5"'), 7.10-7.03 (m, 2H, H-2" and H-6"), 6.97-6.92 (m, 2H, H-3"' and H-5"), $6.61\left(\mathrm{~d}, J=8.4 \mathrm{~Hz}, 1 \mathrm{H}, \mathrm{H}-5^{\prime}\right), 6.29$ (d, $\left.J=2.4 \mathrm{~Hz}, 1 \mathrm{H}, \mathrm{H}-2^{\prime}\right)$, 6.08 (dd, $\left.J=8.4,2.7 \mathrm{~Hz}, 1 \mathrm{H}, \mathrm{H}-6^{\prime}\right), 5.87$ (d, $J=3.6 \mathrm{~Hz}, 2 \mathrm{H}$, H-7'), 4.14-3.93 (m, 2H, H-7), 3.63-3.54 (m, 2H, H-2), 3.463.34 (m, 2H, H-6), 2.57-2.44 (m, 1H, H-4), 2.40-2.30 (m, 1H, $\mathrm{H}-3), 2.21-1.86$ (m, 2H, H-5); ${ }^{13} \mathrm{C} \mathrm{NMR}\left(\mathrm{CDCl}_{3}, 100 \mathrm{MHz}\right)$ : 162.9 (C-1"'), 132.8 (C-4"'), 153.0 (C-1'), 148.0 (C-3'), 141.0 (C-4'), 138.1 (C-4'), 138.0 (C-1"'), 132.4 (C-5"' and 3"'), 129.2 (C-6"' and 2"'), 127.7 (C-2' and 6"'), 115.5 (C-3"' and 5"), 107.0 (C-5'), 105.0 (C-6'), 101.1 (C-7'), 98.0 (C-2'), 68.5 (C-7), 49.4 (C-2), 46.7 (C-6), 43.0 (C-3), 41.0 (C-4), 33.2 ( $\mathrm{C}-5)$; IR (KBr, $\left.v_{\max }, \mathrm{cm}^{-1}\right): 3080(\mathrm{Ar}-\mathrm{H}), 2920(\mathrm{C}-\mathrm{H}), 1570$ (Ar C=C), 1390 (S=O), 1170 (C-F); EIMS m/z: $469[\mathrm{M}]^{+}(5$ $\%), 332(9 \%), 236(2 \%), 191(5 \%), 141(81 \%), 138$ (67\%), $137(35 \%), 121(14 \%), 77(100 \%)$.

$\mathrm{N}$-(4-Toluenesulfonyl)paroxetine (3b): Brown gummy liquid; Yield: $85 \%$; m.f.: $\mathrm{C}_{26} \mathrm{H}_{26} \mathrm{NO}_{5} \mathrm{SF}$; molecular mass: 483; ${ }^{1} \mathrm{H} \mathrm{NMR}\left(\mathrm{CDCl}_{3}, 300 \mathrm{MHz}\right): \delta(\mathrm{ppm}): 7.65(\mathrm{~d}, J=8.4 \mathrm{~Hz}, 2 \mathrm{H}$, H-2"' and H-6"'), 7.34 (d, $J=8.4 \mathrm{~Hz}, 2 \mathrm{H}, \mathrm{H}-3$ "' and H-5"'), 7.07-7.03 (m, 2H, H-2"' and H-6"), 6.96-6.91 (m, 2H, H-3"' and H-5'), 6.58 (d, $\left.J=8.7 \mathrm{~Hz}, 1 \mathrm{H}, \mathrm{H}-5^{\prime}\right), 6.29$ (d, $J=2.4 \mathrm{~Hz}$, $\left.1 \mathrm{H}, \mathrm{H}-2^{\prime}\right), 6.06$ (dd, $\left.J=8.7,2.4 \mathrm{~Hz}, 1 \mathrm{H}, \mathrm{H}-6^{\prime}\right), 5.87$ (s, 2H, H7'), 4.11-3.90 (m, 2H, H-7), 3.59-3.53 (m, 2H, H-2), 3.393.34 (m, 2H, H-6), 2.39 (s, 3H, $\left.\mathrm{CH}_{3}-7^{\prime \prime \prime}\right), 2.32-2.27$ (m, 1H, $\mathrm{H}-3), 2.27-2.22$ (m, 1H, H-4), 1.93-1.82 (m, 2H, H-5); ${ }^{13} \mathrm{C}$ NMR ( $\left.\mathrm{CDCl}_{3}, 100 \mathrm{MHz}\right): 163.9$ (C-7"'), 160.9 (C-1"'), 158.7 (C-4"'), 152.7 (C-1'), 149.1 (C-3'), 140.0 (C-4'), 138.2 (C-1'"), 138.0 (C-4"'), 132.0 (C-3"' and 5"'), 129.0 (C-6"' and 2"'), 128.0 (C-2'" and 6"), 115.9 (C-3'' and 5'), 101.2 (C-7'), 107.2 (C-5'), 105.6 (C-6'), 97.9 (C-2'), 68.7 (C-7), 48.7 (C-2), 45.9 (C-6), 43.0 (C-3), 41.0 (C-4), 33.3 (C-5); IR (KBr, $\left.v_{\max }, \mathrm{cm}^{-1}\right)$ : 3075 (Ar-H), 2915 (C-H), 1579 (Ar C=C), 1393 (S=O), 1181 (C-F); EIMS m/z: $483[\mathrm{M}]^{+}(5 \%), 346(8 \%), 250(10 \%), 236$ (4\%), 191 (6\%), 155 (80\%), 138 (68\%), 137 (33\%), 121 (13\%), $77(100 \%)$.

$\mathrm{N}$-(4-ter-Butylbenzenesulfonyl)paroxetine (3c): White amorphous solid; yield: $95 \%$; m.p.: $240{ }^{\circ} \mathrm{C}$; m.f.: $\mathrm{C}_{29} \mathrm{H}_{32} \mathrm{NO}_{5} \mathrm{SF}$; molecular mass: 525; ${ }^{1} \mathrm{H}$ NMR $\left(\mathrm{CDCl}_{3}, 300\right.$ $\mathrm{MHz}): \delta$ (ppm): 7.71 (d, $J=7.5 \mathrm{~Hz}, 2 \mathrm{H}, \mathrm{H}-2^{\prime \prime \prime}$ and H-6"'), 7.54 (d, $J=7.8 \mathrm{~Hz}, 2 \mathrm{H}, \mathrm{H}-3^{\prime \prime \prime}$ and H-5'"), 7.04-7.02 (m, 2H, H-2" and H-6"), 6.96-6.91 (m, 2H, H-3"' and H-5"), 6.61 (d, $\left.J=8.4 \mathrm{~Hz}, 1 \mathrm{H}, \mathrm{H}-5^{\prime}\right), 6.29$ (s, 1H, H-2'), 6.09 (dd, $J=8.4,2.1$ Hz, 1H, H-6'), 5.86 (s, 2H, H-7'), 4.14-3.39 (m, 2H, H-7), 3.57-3.54 (m, 2H, H-2), 3.39-3.34 (m, 2H, H-6), 2.45- 2.39 (m, 1H, H-3), 2.36-2.32 (m, 1H, H-4), 1.84-1.79 (m, 1H, H5), 1.34 (s, 9H, $\mathrm{CH}_{3}-8^{\prime \prime \prime}, \mathrm{CH}_{3}-9$ "' and $\mathrm{CH}_{3}-10$ "'); ${ }^{13} \mathrm{C}$ NMR $\left(\mathrm{CDCl}_{3}, 100 \mathrm{MHz}\right)$ : 169.2 (C-7"'), 162.7 (C-1"'), 157.5 (C4"'), 152.6 (C-1'), 148.7 (C-3'), 140.2 (C-4'), 138.0 (C-1'), 137.5 (C-4'), 132.0 (C-3"' and 5"'), 129.2 (C-2"' and 6"'), 127.6 (C-2' and 6'), 115.9 (C-3'" and 5'), 101.2 (C-7'), 106.9 (C5'), 105.8 (C-6'), 97.0 (C-2'), 68.3 (C-7), 48.8 (C-2), 46.0 (C6), 43.0 (C-3), 39.8 (C-4), 32.8 (C-5), 25.0 (C-8'", C-9"' and 
C-10"'); IR (KBr, nmax, cm-1): 3083 (Ar-H), 2927 (C-H), 1569 ( $\mathrm{Ar} \mathrm{C}=\mathrm{C}), 1388(\mathrm{~S}=\mathrm{O}), 1167(\mathrm{C}-\mathrm{F}) ; \mathrm{EIMS} m / z: 525[\mathrm{M}]^{+}(5 \%)$, $388(8 \%), 266(24 \%), 240(55 \%), 197(75 \%), 138(68 \%)$, 137 (34\%), 135 (30\%), 133 (60\%), 109 (38\%), 77 (99\%), $56(89 \%)$

$\mathrm{N}$-(2-Mesitylenesulfonyl)paroxetine (3d): Brown gummy liquid; yield: $85 \%$; m.f.: $\mathrm{C}_{28} \mathrm{H}_{30} \mathrm{NO}_{5} \mathrm{SF}$; molecular mass: 511 ; ${ }^{1} \mathrm{H}$ NMR $\left(\mathrm{CDCl}_{3}, 300 \mathrm{MHz}\right): \delta$ (ppm): 7.13-7.08 (m, 2H, H2" and H-6"'), 6.97-6.92 (m, 2H, H-3"' and H-5"), 6.95 (s, 2H, H-3"' and H-5"'), 6.57 (d, $\left.J=8.7 \mathrm{~Hz}, 1 \mathrm{H}, \mathrm{H}-5^{\prime}\right), 6.26(\mathrm{~d}, J=$ $\left.2.4 \mathrm{~Hz}, 1 \mathrm{H}, \mathrm{H}-2^{\prime}\right), 6.04$ (dd, $J=8.4,2.4 \mathrm{~Hz}, 1 \mathrm{H}, \mathrm{H}-6$ '), 5.82 (s, 2H, H-7'), 3.90-3.74 (m, 2H, H-7), 3.57-3.53 (m, 2H, H-2), 3.43-3.37 (m, 2H, H-6), 2.88-2.81 (m, 1H, H-3), 2.64 (s, 6H, $\mathrm{CH}_{3}-7^{\prime \prime \prime}$ and $\left.\mathrm{CH}_{3}-8^{\prime \prime \prime}\right), 2.29$ (s, 3H, $\left.\mathrm{CH}_{3}-9{ }^{\prime \prime}\right), 1.85-1.78(\mathrm{~m}, 1 \mathrm{H}$, $\mathrm{H}-4)$, 1.34-0.81 (m, 2H, H-5); ${ }^{13} \mathrm{C} \mathrm{NMR}\left(\mathrm{CDCl}_{3}, 100 \mathrm{MHz}\right)$ : 163.2 (C-1"'), 161.3 (C-4'"'), 150.0 (C-1'), 147.0 (C-3'), 140.0 (C-4'), 137.6 (C-1"), 137.0 (C-4'"), 131.6 (C-3"' and 5"'), 128.6 (C-2"' and 6"'), 126.5 (C-2' and 6"'), 115.5 (C-3"' and 5"), 107.0 (C-5'), 105.0 (C-6'), 101.2 (C-7'), 97.0 (C-2'), 67.9 (C7), 49.0 (C-2), 46.0 (C-6), 43.0 (C-3), 40.0 (C-4), 35.3 (C-5), 33.7 (C-7'), 33.6 (C-8'), 30.9 (C-9'"); IR (KBr, $\left.v_{\max }, \mathrm{cm}^{-1}\right)$ : 3089 (Ar-H), 2929 (C-H), 1578 (Ar C=C), 1395 (S=O), 1180 (C-F); EIMS m/z: $511[\mathrm{M}]^{+}(4 \%), 374(10 \%), 278(5 \%), 194$ (17\%), 183 (70\%), 138 (55\%), 121 (15\%), 119 (100\%), 77 $(13 \%)$.

$\mathrm{N}$-(4-Acetylbenzenesulfonyl)paroxetine (3e): Brown liquid; yield: $85 \%$; m.f.: $\mathrm{C}_{27} \mathrm{H}_{26} \mathrm{NO}_{6} \mathrm{SF}$; molecular mass: 511; ${ }^{1} \mathrm{H} \mathrm{NMR}\left(\mathrm{CDCl}_{3}, 300 \mathrm{MHz}\right): \delta(\mathrm{ppm}): 8.08(\mathrm{~d}, J=8.4 \mathrm{~Hz}, 2 \mathrm{H}$, H-2"' and H-6"'), 7.87 (d, $J=8.4 \mathrm{~Hz}, 2 \mathrm{H}, \mathrm{H}-3$ "' and H-5"'), 7.12-7.03 (m, 2H, H-2" and H-6"), 6.98-6.88 (m, 2H, H-3'" and $\left.\mathrm{H}-5^{\prime \prime}\right), 6.58\left(\mathrm{~d}, J=8.4 \mathrm{~Hz}, 1 \mathrm{H}, \mathrm{H}-5^{\prime}\right), 6.28(\mathrm{~d}, J=2.4 \mathrm{~Hz}$, $\left.1 \mathrm{H}, \mathrm{H}-2^{\prime}\right), 6.08$ (dd, $J=8.4,2.4 \mathrm{~Hz}, 1 \mathrm{H}, \mathrm{H}-6$ '), 5.86 (d, $J=3.3$ Hz, 2H, H-7'), 4.19-3.95 (m, 2H, H-7), 3.72-3.63 (m, 2H, H2), 3.60-3.37 (m, 2H, H-6), 2.63 (s, 3H, $\mathrm{CH}_{3}-8$ "'), 2.63-2.20 (m, 1H, H-3), 1.89-1.56 (m, 1H, H-4), 1.23-0.81 (m, 2H, H5); ${ }^{13} \mathrm{C} \mathrm{NMR}\left(\mathrm{CDCl}_{3}, 100 \mathrm{MHz}\right): 163.6$ (C-7"'), 162.9 (C-1"'), 159.7 (C-4"'), 153.0 (C-1'), 147.5 (C-3'), 141.0 (C-4'), 138.2 (C-1"), 138.1 (C-4"), 131.9 (C-3"' and 5"'), 129.2 (C-2"' and 6"'), 128.7 (C-2'" and 6"'), 115.6 (C-3"' and 5'"), 101.1 (C-7'), 107.4 (C-5'), 105.6 (C-6'), 97.9 (C-2'), 68.7 (C-7), 49.5 (C-2), 45.7 (C-6), 43.0 (C-3), 41.0 (C-4), 33.6 (C-8"'), 32.7 (C-5); IR (KBr, $\left.v_{\max }, \mathrm{cm}^{-1}\right): 3099(\mathrm{Ar}-\mathrm{H}), 2934(\mathrm{C}-\mathrm{H}), 1720(\mathrm{C}=\mathrm{O})$, 1593 (Ar C=C), $1400(\mathrm{~S}=\mathrm{O}), 1163$ (C-F); EIMS m/z: $511[\mathrm{M}]^{+}$ (3\%), $374(10 \%), 278(4 \%), 138(59 \%), 191(7 \%), 183(52$ $\%), 121(20 \%), 141(75 \%), 43(40 \%), 77$ (100\%).

$\mathrm{N}$-(4-Methoxybenzenesulfonyl)paroxetine (3f): White amorphous solid; yield: $90 \%$; m.p.: $215^{\circ} \mathrm{C}$; m.f.: $\mathrm{C}_{26} \mathrm{H}_{26} \mathrm{NO}_{6} \mathrm{SF}$; molecular mass: 499; ${ }^{1} \mathrm{H} \mathrm{NMR}\left(\mathrm{CDCl}_{3}, 300 \mathrm{MHz}\right): \delta(\mathrm{ppm})$ : $7.70\left(\mathrm{~d}, J=8.4 \mathrm{~Hz}, 2 \mathrm{H}, \mathrm{H}-2\right.$ "' $^{\prime}$ and H-6"'), 7.36 (d, $J=8.4 \mathrm{~Hz}$, 2H, H-3"' and H-5"'), 7.08-7.03 (m, 2H, H-2'" and H-6'), 6.986.91 (m, 2H, H-3"' and H-5'), 6.61 (d, $\left.J=8.4 \mathrm{~Hz}, 1 \mathrm{H}, \mathrm{H}-5^{\prime}\right)$, $6.29\left(\mathrm{~d}, J=2.1 \mathrm{~Hz}, 1 \mathrm{H}, \mathrm{H}-2{ }^{\prime}\right), 6.09$ (dd, $J=8.4,2.4 \mathrm{~Hz}, 1 \mathrm{H}, \mathrm{H}-$ 6'), 5.85 (s, 2H, H-7'), 4.09-3.92 (m, 2H, H-7), 3.57-3.54 (m, 2H, H-2), 3.39-3.34 (m, 2H, H-6), 2.41 (s, 3H, $\mathrm{CH}_{3}-7$ "'), 2.342.30 (m, 1H, H-3), 2.26-2.20 (m, 1H, H-4) 1.93-1.87 (m, 2H, $\mathrm{H}-5) ;{ }^{13} \mathrm{C} \mathrm{NMR}\left(\mathrm{CDCl}_{3}, 100 \mathrm{MHz}\right): 162.9$ (C-1"'), 158.8 (C-4"'), 152.5 (C-1'), 149.0 (C-3'), 140.2 (C-4'), 138.1 (C-1'"), 138.0 (C-4") 132.0 (C-3"' and 5"'), 129.8 (C-2"' and 6"'), 128.0 (C-2' and 6"), 115.9 (C-3' and 5'), 101.2 (C-7'), 106.9
(C-5'), 105.8 (C-6'), 96.8 (C-2'), 68.3 (C-7), 48.9 (C-2), 46.0 (C-6), 43.0 (C-3), 39.9 (C-4), 33.0 (C-7"'), 32.8 (C-5); IR (KBr, $\left.V_{\max }, \mathrm{cm}^{-1}\right): 3078(\mathrm{Ar}-\mathrm{H}), 2920(\mathrm{C}-\mathrm{H}), 1573(\mathrm{Ar} \mathrm{C}=\mathrm{C}), 1385$ $(\mathrm{S}=\mathrm{O}), 1167$ (C-F); EIMS m/z: 499 [M] $^{+}$(3\%), $362(12 \%)$, $241(31 \%), 215$ (60\%), 172 (80\%), $138(65 \%), 137$ (40\%), 135 (45\%), $121(15 \%), 109$ (25\%), 77 (100\%).

$\mathrm{N}$-(4-Acetamidobenzenesulfonyl)paroxetine (3g): White amorphous solid; yield: $75 \%$; m.p.: $200{ }^{\circ} \mathrm{C}$; m.f.: $\mathrm{C}_{27} \mathrm{H}_{27} \mathrm{~N}_{2} \mathrm{O}_{6} \mathrm{SF}$; molecular mass: 526; ${ }^{1} \mathrm{H} \mathrm{NMR}\left(\mathrm{CDCl}_{3}, 300 \mathrm{MH}_{\mathrm{z}}\right): \delta(\mathrm{ppm})$ : $7.73\left(\mathrm{~d}, J=8.4 \mathrm{~Hz}, 2 \mathrm{H}, \mathrm{H}-3{ }^{\prime \prime \prime}\right.$ and H-5"'), 7.69 (d, $J=8.4 \mathrm{~Hz}$, 2H, H-2"' and H-6"'), 7.14-7.04 (m, 2H, H-2'" and H-6'"), 7.006.92 (m, 2H, H-3"' and H-5"), 6.58 (d, $J=8.4 \mathrm{~Hz}, 1 \mathrm{H}, \mathrm{H}-5$ '), 6.29 (d, $\left.J=1.5 \mathrm{~Hz}, 1 \mathrm{H}, \mathrm{H}-2^{\prime}\right), 6.09$ (dd, $J=8.4,1.5 \mathrm{~Hz}, 1 \mathrm{H}$, H-6'), 5.86 (d, J = 3.6 Hz, 2H, H-7'), 4.10-3.90 (m, 2H, H-7), 3.63-3.54 (m, 2H, H-2), 3.48-3.34 (m, 2H, H-6), 2.55-2.43 (m, 1H, H-4), 2.39-2.29 (m, 1H, H-3), 2.08-1.88 (m, 2H, H5); ${ }^{13} \mathrm{C} \mathrm{NMR}\left(\mathrm{CDCl}_{3}, 100 \mathrm{MHz}\right): 164.0$ (C-7"'), 162.9 (C-1"'), 159.5 (C-4"'), 152.9 (C-1'), 148.0 (C-3'), 141.0 (C-4'), 138.1 (C-1'"), 138.0 (C-4'), 131.9 (C-3"' and 5"'), 129.2 (C-2"' and 6"'), 128.7 (C-2'" and 6'), 115.5 (C-3"' and 5'), 107.2 (C-5'), 105.6 (C-6'), 101.2 (C-7'), 97.9 (C-2'), 68.7 (C-7), 49.5 (C-2), 45.8 (C-6), 43.0 (C-3), 41.0 (C-4), 33.3 (C-5), 30.9 (C-8"'); IR $\left(\mathrm{KBr}, \mathrm{v}_{\max }, \mathrm{cm}^{-1}\right): 3076(\mathrm{Ar}-\mathrm{H}), 2914(\mathrm{C}-\mathrm{H}), 1649(\mathrm{C}=\mathrm{O})$, $1581(\mathrm{Ar} \mathrm{C}=\mathrm{C}), 1399(\mathrm{~S}=\mathrm{O}), 1189(\mathrm{C}-\mathrm{F})$; EIMS m/z: $526[\mathrm{M}]^{+}$ (3\%), $389(5 \%), 328(4 \%), 138(74 \%), 137$ (36\%), $121(12$ $\%), 198$ (100\%), 176 (6\%), $81(10 \%), 79(12 \%)$.

$\mathrm{N}$-(4-Chlorobenzenesulfonyl)paroxetine (3h): White amorphous solid; yield: $90 \%$; m.p.: $210^{\circ} \mathrm{C}$; m.f.: $\mathrm{C}_{25} \mathrm{H}_{23} \mathrm{NO}_{5} \mathrm{SClF}$; molecular mass: $503 ;{ }^{1} \mathrm{H} \mathrm{NMR}\left(\mathrm{CDCl}_{3}, 300 \mathrm{MHz}\right): \delta(\mathrm{ppm})$ : $7.73\left(\mathrm{~d}, J=8.4 \mathrm{~Hz}, 2 \mathrm{H}, \mathrm{H}-2\right.$ '"' $^{2} \mathrm{nd}$ H-6"'), 7.53 (d, $J=8.4 \mathrm{~Hz}$, 2H, H-3"' and H-5"'), 7.08-7.04 (m, 2H, H-2'" and H-6"'), 6.976.92 (m, 2H, H-3"' and H-5'), 6.61 (d, $\left.J=8.4 \mathrm{~Hz}, 1 \mathrm{H}, \mathrm{H}-5^{\prime}\right)$, 6.30 (d, $J=2.4 \mathrm{~Hz}, 1 \mathrm{H}, \mathrm{H}-2$ '), 6.09 (dd, $J=8.1,2.4 \mathrm{~Hz}, 1 \mathrm{H}$, H-6'), 5.88 (s, 2H, H-7'), 4.11-3.90 (m, 2H, H-7), 3.58-3.54 (m, 2H, H-2), 3.40-3.34 (m, 2H, H-6), 2.51-2.42 (m, 1H, H4), 2.37-2.30 (m, 1H, H-3), 1.94-1.85 (m, 2H, H-5); ${ }^{13} \mathrm{C} \mathrm{NMR}$ $\left(\mathrm{CDCl}_{3}, 100 \mathrm{MHz}\right): 163.0$ (C-1"'), 160.5 (C-4"'), 153.9 (C-1'), 148.0 (C-3'), 141.0 (C-4'), 138.1 (C-1'), 138.0 (C-4'"), 132.4 (C-3"' and 5"'), 129.2 (C-2"' and 6"'), 128.7 (C-2'" and 6"), 115.5 (C-3'" and 5'), 101.2 (C-7'), 107.0 (C-5'), 105.0 (C-6'), 97.9 (C-2'), 68.5 (C-7), 49.5 (C-2), 46.7 (C-6), 43.0 (C-3), 41.0 (C-4), 33.3 (C-5); IR (KBr, $\left.v_{\max }, \mathrm{cm}^{-1}\right): 3091$ (Ar-H), 2931 (C-H), 1596 (Ar C=C), 1397 (S=O), 1185 (C-F), 693 (C-Cl); EIMS m/z: $503[\mathrm{M}]^{+}(5 \%), 366(15 \%), 245$ (30\%), 219 (56\%), 176 (80\%), 138 (70\%), 137 (40\%), 135 (25\%), 121 (20\%), $109(37 \%), 77$ (99\%).

$\mathrm{N}$-(4-Bromobenzenesulfonyl)paroxetine (3i): White amorphous solid; yield: $90 \%$; m.p.: $205^{\circ} \mathrm{C}$; m.f.: $\mathrm{C}_{25} \mathrm{H}_{23} \mathrm{NO}_{5} \mathrm{SBrF}$; molecular mass: $548 ;{ }^{1} \mathrm{H}$ NMR $\left(\mathrm{CDCl}_{3}, 300 \mathrm{MHz}\right): \delta(\mathrm{ppm})$ : $7.69\left(\mathrm{~d}, J=8.7 \mathrm{~Hz}, 2 \mathrm{H}, \mathrm{H}-2\right.$ "' $^{\prime}$ and H-6"'), 7.62 (d, $J=8.7 \mathrm{~Hz}$, 2H, H-3"' and H-5"'), 7.08-7.04 (m, 2H, H-2'" and H-6"'), 6.976.92 (m, 2H, H-3"' and H-5"), 6.59 (d, $\left.J=8.4 \mathrm{~Hz}, 1 \mathrm{H}, \mathrm{H}-5^{\prime}\right)$, 6.29 (d, $J=2.4 \mathrm{~Hz}, 1 \mathrm{H}, \mathrm{H}-2$ '), 6.07 (dd, $J=8.4,2.4 \mathrm{~Hz}, 1 \mathrm{H}$, H-6'), 5.86 (s, 2H, H-7'), 4.10-3.90 (m, 2H, H-7), 3.63-3.54 (m, 2H, H-2), 3.48-3 (m, 2H, H-6), 2.55-2.43 (m, 1H, H-4), 2.39-2.29 (m, 1H, H-3), 2.08-1.88 (m, 2H, H-5); ${ }^{13} \mathrm{C}$ NMR $\left(\mathrm{CDCl}_{3}, 100 \mathrm{MHz}\right): 163.0$ (C-1"'), 160.5 (C-4"'), 153.9 (C-1'), 148.0 (C-3'), 141.0 (C-4'), 138.1 (C-1'"), 138.0 (C-4'), 132.4 (C-3"' and 5"'), 129.2 (C-2"' and 6"'), 128.7 (C-2'" and 6"), 
115.5 (C-3' ' and 5'), 101.2 (C-7'), 107.0 (C-5'), 105.0 (C-6'), 97.9 (C-2'), 68.5 (C-7), 49.5 (C-2), 46.7 (C-6), 43.0 (C-3), 41.0 (C-4), 33.3 (C-5); IR (KBr, $\left.v_{\max }, \mathrm{cm}^{-1}\right): 3069$ (Ar-H), 2937 (C-H), 1591 (Ar C=C), $1401(\mathrm{~S}=\mathrm{O}), 1201(\mathrm{C}-\mathrm{F})$; EIMS m/z: $548[\mathrm{M}]^{+}(3 \%), 412(3 \%), 328(2 \%), 290(8 \%), 262(15 \%)$, 219 (26\%), 155 (44\%), 138 (67\%), 76 (100\%).

$\mathrm{N}$-(2,3-Dichlorobenzenesulfonyl)paroxetine (3j): White amorphous solid; yield: $90 \%$; m.p.: $215^{\circ} \mathrm{C}$; m.f.: $\mathrm{C}_{25} \mathrm{H}_{22} \mathrm{NO}_{5} \mathrm{SFCl}_{2}$; molecular mass: 537; ${ }^{1} \mathrm{H} \mathrm{NMR}\left(\mathrm{CDCl}_{3}, 300\right.$ $\mathrm{MHz}): \delta(\mathrm{ppm}): 8.06$ (dd, $J=6.9,2.3 \mathrm{~Hz}, 1 \mathrm{H}, \mathrm{H}-6 " '), 7.68$ (dd, $\left.J=6.9,2.4 \mathrm{~Hz}, 1 \mathrm{H}, \mathrm{H}-4{ }^{\prime \prime \prime}\right), 7.36$ (t, $\left.J=8.1 \mathrm{~Hz}, 1 \mathrm{H}, \mathrm{H}-5^{\prime \prime}\right)$, 7.13-7.08 (m, 2H, H-2"' and H-6"'), 6.98-6.93 (m, 2H, H-3"' and H-5"'), 6.61 (d, $\left.J=8.4 \mathrm{~Hz}, 1 \mathrm{H}, \mathrm{H}-5^{\prime}\right), 6.30(\mathrm{~d}, J=2.4$ Hz, 1H, H-2'), 6.10 (dd, J = 8.1, 2.1 Hz, 1H, H-6'), 5.86 (s, 2H, H-7'), 4.12-3.99 (m, 2H, H-7), 3.58 (m, 2H, H-2), 3.43-3.38 (m, 2H, H-6), 2.96-2.85 (m, 1H, H-3), 2.70-2.61 (m, 1H, H4), 1.87-1.79 (m, 2H, H-5); ${ }^{13} \mathrm{C} \mathrm{NMR}\left(\mathrm{CDCl}_{3}, 100 \mathrm{MHz}\right): 163.5$ (C-1"'), 159.3 (C-2"'), 157.6 (C-3"'), 153.0 (C-1'), 148.2 (C3'), 140.9 (C-4'), 138.2 (C-4'), 138.0 (C-1'), 132.8 (C-4"'), 132.4 (C-5"'), 129.2 (C-6"'), 125.6 (C-2'" and 6"'), 115.4 (C-3'" and 5'), 101.1 (C-7'), 107.2 (C-5'), 104.9 (C-6'), 97.9 (C-2'), 68.5 (C-7), 49.5 (C-2), 46.5 (C-6), 43.0 (C-3), 41.0 (C-4), 33.1 (C-5); IR (KBr, $\left.v_{\max }, \mathrm{cm}^{-1}\right)$ : $3090(\mathrm{Ar}-\mathrm{H}), 2941(\mathrm{C}-\mathrm{H})$, 1584 (Ar C=C), 1410 (S=O), 1163 (C-F), 685 (C-Cl); EIMS $\mathrm{m} / \mathrm{z}: 537[\mathrm{M}]^{+}(3 \%), 400(10 \%), 280(30 \%), 254(55 \%), 211$ (87\%), 147 (95\%), 138 (70\%), 137 (45\%), 135 (30\%), 109 (38\%).

$\mathrm{N}$-(3,4-Dichlorobenzenesulfonyl)paroxetine (3k): White amorphous solid; Yield: $90 \%$; m.p.: $215^{\circ} \mathrm{C}$; m.f.: $\mathrm{C}_{25} \mathrm{H}_{22} \mathrm{NO}_{5} \mathrm{SFCl}_{2}$; molecular mass: $537 ;{ }^{1} \mathrm{H}$ NMR $\left(\mathrm{CDCl}_{3}, 300 \mathrm{MHz}\right): \delta(\mathrm{ppm})$ : 7.87 (s, 1H, H-2"'), 7.61 (s, 2H, H-5"' and H-6"'), 7.09-7.05 (m, 2H, H-2" and H-6"), 6.98-6.92 (m, 2H, H-3"' and H-5"), $6.61\left(\mathrm{~d}, J=8.4 \mathrm{~Hz}, 1 \mathrm{H}, \mathrm{H}-5^{\prime}\right), 6.31$ (d, $\left.J=2.4 \mathrm{~Hz}, 1 \mathrm{H}, \mathrm{H}-2^{\prime}\right)$, $6.10\left(\mathrm{dd}, J=8.4,2.1 \mathrm{~Hz}, 1 \mathrm{H}, \mathrm{H}-6^{\prime}\right), 5.86$ (s, 2H, H-7'), 4.11 3.92 (m, 2H, H-7), 3.59-3.55 (m, 2H, H-2), 3.41-3.36 (m, 2H, H-6), 2.50-2.42 (m, 1H, H-3), 2.39-2.35 (m, 1H, H-4), 1.951.88 (m, 2H, H-5); ${ }^{13} \mathrm{C}$ NMR $\left(\mathrm{CDCl}_{3}, 100 \mathrm{MHz}\right): 163.5(\mathrm{C}-$ 1"'), 159.2 (C-4'"), 157.5 (C-3'"'), 153.5 (C-1'), 147.8 (C-3'), 140.8 (C-4'), 138.2 (C-4'"), 137.6 (C-1'), 133.2 (C-2"'), 132.4 (C-5"'), 130.1 (C-6"'), 125.7 (C-2'" and 6'), 115.4 (C-3'" and 5'), 101.1 (C-7'), 107.2 (C-5'), 105.0 (C-6'), 97.8 (C-2'), 68.5 (C-7), 49.6 (C-2), 46.6 (C-6), 43.0 (C-3), 41.0 (C-4), 33.1 (C5); IR (KBr, $\left.v_{\max }, \mathrm{cm}^{-1}\right): 3092(\mathrm{Ar}-\mathrm{H}), 2943(\mathrm{C}-\mathrm{H}), 1586(\mathrm{Ar}$ $\mathrm{C}=\mathrm{C}), 1408$ (S=O), 1165 (C-F), 689 (C-Cl); EIMS m/z: 537 $[\mathrm{M}]^{+}(3 \%), 400(10 \%), 280(30 \%), 254(55 \%), 211(87 \%)$, 147 (95\%), 138 (70\%), 137 (45\%), 135 (30\%), 109 (38\%).

$\mathrm{N}$-(Benzylsulphonyl)paroxetine (31): Brown liquid; yield: $85 \%$; m.f.: $\mathrm{C}_{26} \mathrm{H}_{26} \mathrm{NO}_{5} \mathrm{SF}$; molecular mass: 483; ${ }^{1} \mathrm{H}$ NMR $\left(\mathrm{CDCl}_{3}, 300 \mathrm{MHz}\right): \delta$ (ppm): 7.97 (d, $J=8.4 \mathrm{~Hz}, 2 \mathrm{H}, \mathrm{H}-2^{\prime \prime \prime}$ and H-6"'), 7.79-7.76 (m, 1H, H-4"'), 7.68-7.58 (m, 2H, H-3"' and H-5"'), 7.10-7.08 (m, 2H, H-2'" and H-6'"), 6.96-6.89 (m, 2H, H-3"' and H-5"), 6.57 (d, $J=8.4 \mathrm{~Hz}, 1 \mathrm{H}, \mathrm{H}-5$ '), 6.27 (d, $\left.J=2.4 \mathrm{~Hz}, 1 \mathrm{H}, \mathrm{H}-2^{\prime}\right), 6.05$ (dd, $\left.J=8.4,2.4 \mathrm{~Hz}, 1 \mathrm{H}, \mathrm{H}-6^{\prime}\right)$, 5.85 (d, $\left.J=3.3 \mathrm{~Hz}, 2 \mathrm{H}, \mathrm{H}-7^{\prime}\right), 4.89$ (br.s, 2H, H-7'"), 4.254.00 (m, 2H, H-7), 3.72-3.53 (m, 2H, H-2), 3.44-3.36 (m, 2H, H-6), 2.64-2.27 (m, 1H, H-3), 1.98-1.83 (m, 1H, H-4), 1.83$0.83(\mathrm{~m}, 2 \mathrm{H}, \mathrm{H}-5) ;{ }^{13} \mathrm{C} \mathrm{NMR}\left(\mathrm{CDCl}_{3}, 100 \mathrm{MHz}\right): 162.7$ (C7"'), 162.8 (C-1"'), 159.2 (C-4"'), 153.0 (C-1'), 149.3 (C-3'), 140.0 (C-4'), 138.2 (C-1'"), 137.9 (C-4'), 133.0 (C-3"' and
5"'), 129.0 (C-2"' and 6"'), 128.0 (C-2' and 6"), 115.9 (C-3"' and 5"), 107.2 (C-5'), 105.6 (C-6'), 101.2 (C-7'), 97.9 (C-2'), 68.7 (C-7), 48.7 (C-2), 45.9 (C-6), 43.0 (C-3), 41.0 (C-4), 33.3 (C-5); IR (KBr, $\left.v_{\max }, \mathrm{cm}^{-1}\right)$ : 3096 (Ar-H), 2923 (C-H), 1577 ( $\mathrm{ArC}=\mathrm{C}), 1391(\mathrm{~S}=\mathrm{O}), 1178(\mathrm{C}-\mathrm{F}) ; \mathrm{EIMS} \mathrm{m} / z: 483[\mathrm{M}]^{+}$ (3\%), 346 (8\%), 250.0 (4\%), 138 (60\%), 137 (25\%), 155 (70 \%), 91 (40\%), $77(100 \%), 121$ (10\%), 91 (4\%).

$\mathrm{N}$-(Naphthalene-1-ylsulfonyl)paroxetine (3m): Brown gummy liquid; Yield: $85 \%$; m.f.: $\mathrm{C}_{29} \mathrm{H}_{26} \mathrm{NO}_{5} \mathrm{SF}$; molecular mass: $519 ;{ }^{1} \mathrm{H} \mathrm{NMR}\left(\mathrm{CDCl}_{3}, 300 \mathrm{MHz}\right): \delta(\mathrm{ppm}): 8.76(\mathrm{~d}, J=$ $\left.8.7 \mathrm{~Hz}, 1 \mathrm{H}, \mathrm{H}-8^{\prime \prime \prime}\right), 8.26$ (dd, $\left.J=7.2,1.0 \mathrm{~Hz}, 1 \mathrm{H}, \mathrm{H}-2^{\prime \prime \prime}\right), 8.07$ (d, $\left.J=8.1 \mathrm{~Hz}, 1 \mathrm{H}, \mathrm{H}-4{ }^{\prime \prime}\right), 7.94$ (d, $\left.J=7.8 \mathrm{~Hz}, 1 \mathrm{H}, \mathrm{H}-5^{\prime \prime}\right)$, 7.70-7.66 (m, 1H, H-7"'), 7.65-7.58 (m, 1H, H-6"'), 7.57-7.49 (m, 1H, H-3"'), 7.02-6.99 (m, 2H, H-2"' and H-6"), 6.95-6.88 (m, 2H, H-3'" and H-5'), 6.56 (d, J = 8.4 Hz, 1H, H-5'), 6.24 (d, $\left.J=2.4 \mathrm{~Hz}, 1 \mathrm{H}, \mathrm{H}-2^{\prime}\right), 6.03$ (dd, $J=8.4,2.4 \mathrm{~Hz}, 1 \mathrm{H}, \mathrm{H}-6$ '), 5.86 (s, 2H, H-7'), 4.21-4.01 (m, 2H, H-7), 3.54-3.50 (m, 2H, H-2), 3.37-3.32 (m, 2H, H-6), 2.69-2.63 (m, 1H, H-3), 1.831.59 (m, 1H, H-4), 1.28-0.81 (m, 2H, H-5) ${ }^{13} \mathrm{C}-\mathrm{NMR}\left(\mathrm{CDCl}_{3}\right.$, $100 \mathrm{MHz}): 153.9$ (C-1'), 148.2 (C-3'), 141.9 (C-4'), 138.2 (C1"), 138.1 (C-4"'), 135.6 (C-6"'), 135.1 (C-2"'), 134.7 (C-10"'), 134.4 (C-4"'), 132.0 (C-1"'), 131.7 (C-5"'), 131.2 (C-9"'), 128.7 (C-2' and 6"), 126.1 (C-7"'), 125.2 (C-3"'), 124.0 (C-8"'), 115.8 (C-3"' and 5'), 107.8 (C-5'), 105.6 (C-6'), 101.2 (C-7'), 97.9 (C-2'), 68.5 (C-7), 49.5 (C-2), 46.7 (C-6), 43.1 (C-3), 41.8 (C4), 33.3 (C-5); IR (KBr, $\left.v_{\max }, \mathrm{cm}^{-1}\right): 3105$ (Ar-H), 2951 (C-H), 1602 (Ar C=C), $1403(\mathrm{~S}=\mathrm{O}), 1211$ (C-F); EIMS m/z: 519 [M] ${ }^{+}$ (6\%), 382 (12\%), 286 (9\%), 234 (54\%), 109 (40\%), 191 (80\%), 138 (64\%), 127 (99\%), 121 (14\%).

$\mathrm{N}$-(Naphthalene-2-ylsulfonyl)paroxetine (3n): Brown gummy liquid; yield: $85 \%$; m.f.: $\mathrm{C}_{29} \mathrm{H}_{26} \mathrm{NO}_{5} \mathrm{SF}$; molecular mass: $519 ;{ }^{1} \mathrm{H} \mathrm{NMR}\left(\mathrm{CDCl}_{3}, 400 \mathrm{MHz}\right): \delta(\mathrm{ppm}): 8.36$ (s, $1 \mathrm{H}$, H-1"'), 7.99 (d, $J=8.4$ Hz, 2H, H-8"'and H-3"'), 7.93 (d, $J=$ $\left.8.0 \mathrm{~Hz}, 1 \mathrm{H}, \mathrm{H}-44^{\prime \prime}\right), 7.77$ (dd, $\left.J=8.8,1.6 \mathrm{~Hz}, 1 \mathrm{H}, \mathrm{H}-5{ }^{\prime \prime}\right), 7.67-$ 7.61 (m, 2H, H-6"'and H-7"'), 7.04 (d, $J=8.4$ Hz, 2H, H-2'" and H-6"'), 6.95 (d, $J=8.8 \mathrm{~Hz}, 2 \mathrm{H}, \mathrm{H}-3$ "' and H-5"'), 6.57 (d, $\left.J=8.4 \mathrm{~Hz}, 1 \mathrm{H}, \mathrm{H}-5^{\prime}\right), 6.28$ (d, $\left.J=2.4 \mathrm{~Hz}, 1 \mathrm{H}, \mathrm{H}-2^{\prime}\right), 6.07$ (dd, $J=8.4,2.8 \mathrm{~Hz}, 1 \mathrm{H}, \mathrm{H}-6 '), 5.86$ (s, 2H, H-7'), 4.21-4.01 (m, 2H, H-7), 3.54-3.50 (m, 2H, H-2), 3.37-3.32 (m, 2H, H-6), 2.69-2.63 (m, 1H, H-3), 1.83-1.59 (m, 1H, H-4), 1.28-0.81 (m, 2H, H-5); ${ }^{13} \mathrm{C} \mathrm{NMR}\left(\mathrm{CDCl}_{3}, 100 \mathrm{MHz}\right): 153.9(\mathrm{C}-1$ '), 148.2 (C-3'), 141.9 (C-4'), 138.2 (C-1'), 138.1 (C-4'), 137.1 (C5"'), 137.0 (C-9"'), 136.9 (C-2"'), 136.0 (C-7"'), 130.4 (C-10"'), 129.7 (C-8"'), 129.0 (C-4"'), 128.7 (C-2' and 6"), 127.8 (C6"'), 125.3 (C-1'"), 123.4 (C-3"'), 115.8 (C-3"' and 5'), 107.8 (C-5'), 105.6 (C-6'), 101.2 (C-7'), 97.9 (C-2'), 68.5 (C-7), 49.5 (C-2), 46.7 (C-6), 43.1 (C-3), 41.8 (C-4), 33.3 (C-5); IR (KBr, $\left.V_{\max }, \mathrm{cm}^{-1}\right): 3106(\mathrm{Ar}-\mathrm{H}), 2950(\mathrm{C}-\mathrm{H}), 1601$ (Ar C=C), 1404 $(\mathrm{S}=\mathrm{O}), 1213$ (C-F); EIMS m/z: 519 [M] $^{+}(6 \%), 382(12 \%)$, 286 (9\%), 234 (54\%),109 (40\%), 191 (80\%), 138 (64\%), $127(100 \%), 121(14 \%)$.

\section{RESULTS AND DISCUSSION}

Paroxetine is already being used in the market as drug and we have made an attempt to evaluate its enzyme inhibition activity. In the present research work, a number of $\mathrm{N}$-aralkyl/ aryl sulfonated derivatives of paroxetine were synthesized by the coupling of different sulfonyl chlorides, 2a-n, with paroxetine (1) under an alkaline $\mathrm{pH}$ in aqueous media 
(Scheme-I). The synthesized compounds were screened against lipoxygenase enzyme to search out their enzyme inhibition activity. The maximum yields of products, 3a-n, were obtained within $3 \mathrm{~h}$ by continuous stirring at RT. The products were isolated by filtration after the addition of dil. $\mathrm{HCl}$ and washing of precipitates was carried out with cold distilled water ${ }^{13,14}$. The resulting products were used for the spectral analysis and enzyme inhibition activity after recrystallization from methanol. Paroxetine was white amorphous powder and named as 3-\{(benzo[1,3]dioxol-5-yloxy)methyl $\}$ 4-(4-fluorophenyl)-1-(phenylsulfonyl)piperidine. The stereochemistry of the paroxetine was designed by the comparison of ${ }^{1} \mathrm{H}$ NMR coupling constant and also the ${ }^{13} \mathrm{C}$ NMR signals with the literature ${ }^{15}$.

The synthesized compound $\mathbf{3 a}$, obtained as white amorphous solid having $85 \%$ yield and $205^{\circ} \mathrm{C}$ melting point, had the molecular formula $\mathrm{C}_{25} \mathrm{H}_{24} \mathrm{NO}_{5} \mathrm{SF}$ established by EI-MS showing $[\mathrm{M}]^{+}$peak at $\mathrm{m} / \mathrm{z} 469$ and also by counting the number of protons and carbons in ${ }^{1} \mathrm{H}$ and ${ }^{13} \mathrm{C}$ NMR spectrum, respectively. The IR spectrum showed the signal of a sulfonyl group $\left(1390 \mathrm{~cm}^{-1}\right)$. The other major peaks appeared at 3080, 2920, 1570 and $1170 \mathrm{~cm}^{-1}$, due to stretching of aromatic $\mathrm{C}-\mathrm{H}$, aliphatic $\mathrm{C}-\mathrm{H}$, aromatic $\mathrm{C}=\mathrm{C}$ and carbon-flourine bond, respectively in the molecule. The EI-MS presented two distinct peaks at $\mathrm{m} / \mathrm{z}$ 121 and 141 attributed to the presence of benzodioxane and benzenesulfonyl moiety, respectively. The other prominent peaks are clearly mentioned in the mass fragmentation pattern of this molecule in Fig. 1. In the aromatic region of the ${ }^{1} \mathrm{H}$
NMR spectrum, the signals appeared at $\delta 7.78(\mathrm{dd}, J=6.9$, $1.5 \mathrm{~Hz}, 2 \mathrm{H}, \mathrm{H}-2^{\prime \prime \prime}$ and H-6"'), 7.63-7.61 (m, 1H, H-4"') and 7.58 (dd, $J=6.9,1.5 \mathrm{~Hz}, 2 \mathrm{H}, \mathrm{H}-3^{\prime \prime \prime}$ and H-5"') which were assigned to the mono-substituted benzenesulfonyl ring. The signal resonated at $\left.d 6.61(\mathrm{~d}, J=8.4 \mathrm{~Hz}, 1 \mathrm{H}, \mathrm{H}-5)^{\prime}\right), 6.29(\mathrm{~d}$, $\left.J=2.4 \mathrm{~Hz}, 1 \mathrm{H}, \mathrm{H}-2^{\prime}\right), 6.08$ (dd, $\left.J=8.4,2.7 \mathrm{~Hz}, 1 \mathrm{H}, \mathrm{H}-6^{\prime}\right)$ and 5.87 (d, $\left.J=3.6 \mathrm{~Hz}, 2 \mathrm{H}, \mathrm{H}-7^{\prime}\right)$ which were corroborated the presence of benzo[1,3]dioxol moiety in the compound. Similarly, the signals showed at $\delta 7.10-7.03\left(\mathrm{~m}, 2 \mathrm{H}, \mathrm{H}-2^{\prime \prime}\right.$ and H-6") and 6.97-6.92 (m, 2H, H-3" and H-5"') which indicated the presence of para di-substituted phenyl group. In the aliphatic region, the signal appeared at $\delta 4.14-3.93(\mathrm{~m}, 2 \mathrm{H}$, $\mathrm{H}-7)$ which was assign to the methane proton and the signal at א 3.63-3.54 (m, 2H, H-2), 3.46-3.34 (m, 2H, H-6), 2.57-2.44 (m, $1 \mathrm{H}, \mathrm{H}-4), 2.40-2.30$ (m, 1H, H-3) and 2.21-1.86 (m, 2H, H-5) which were given to the piperidine ring. In ${ }^{13} \mathrm{C} \mathrm{NMR}$ spectrum (BB and DEPT) 25 signals appeared, disclosing the presence of six quaternary carbons, fourteen methine carbons and five methylene carbons. The substituted phenyl sulfonyl ring signals appeared at $\delta_{\mathrm{C}}(\mathrm{ppm}) 132.8$ (C-4"'), 132.4 (C-5"' and 3"') and 129.2 (C-6"' and 2 "') for five methine carbons with intensities of 1:2:2, respectively and its quaternary carbon signal appeared at $\delta_{\mathrm{C}}(\mathrm{ppm}) 1$ 62.9 (C-1"'). The remaining signals of paroxetine moiety were mentioned in the experimental section and also compare with the literature ${ }^{17-19}$. On the basis of all above data, the structure of compound 3a was named as $N$-(benzenesulfonyl)paroxetine. Similarly, the structures of other compounds $\mathbf{3 b}$ b-n were characterized by ${ }^{1} \mathrm{H} \mathrm{NMR},{ }^{13} \mathrm{C}$ NMR, IR and EI-MS.

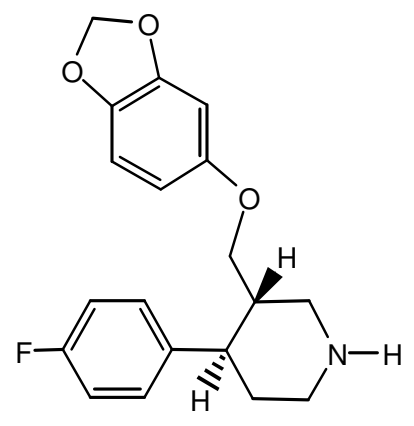

1

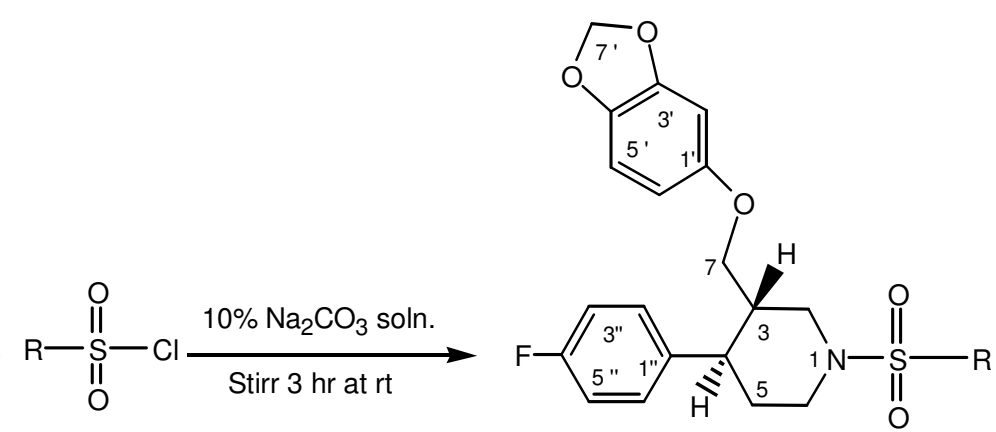

2a-n

3a-n

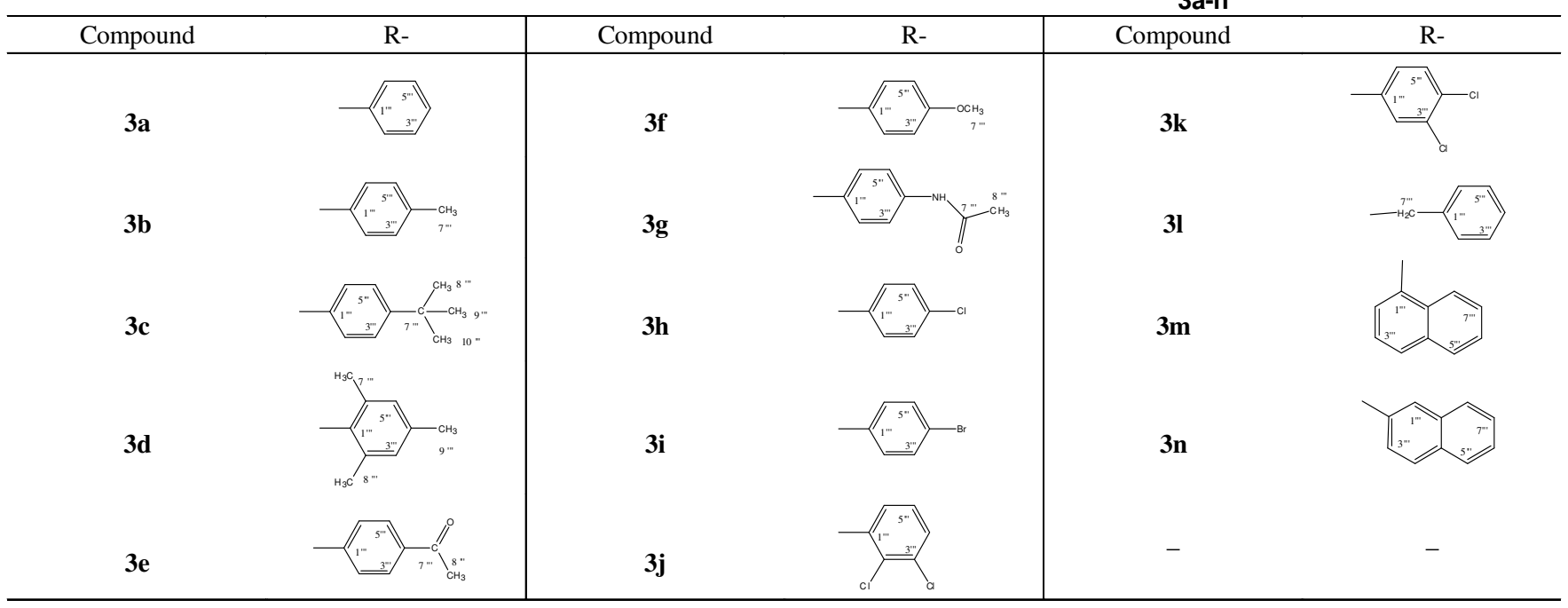




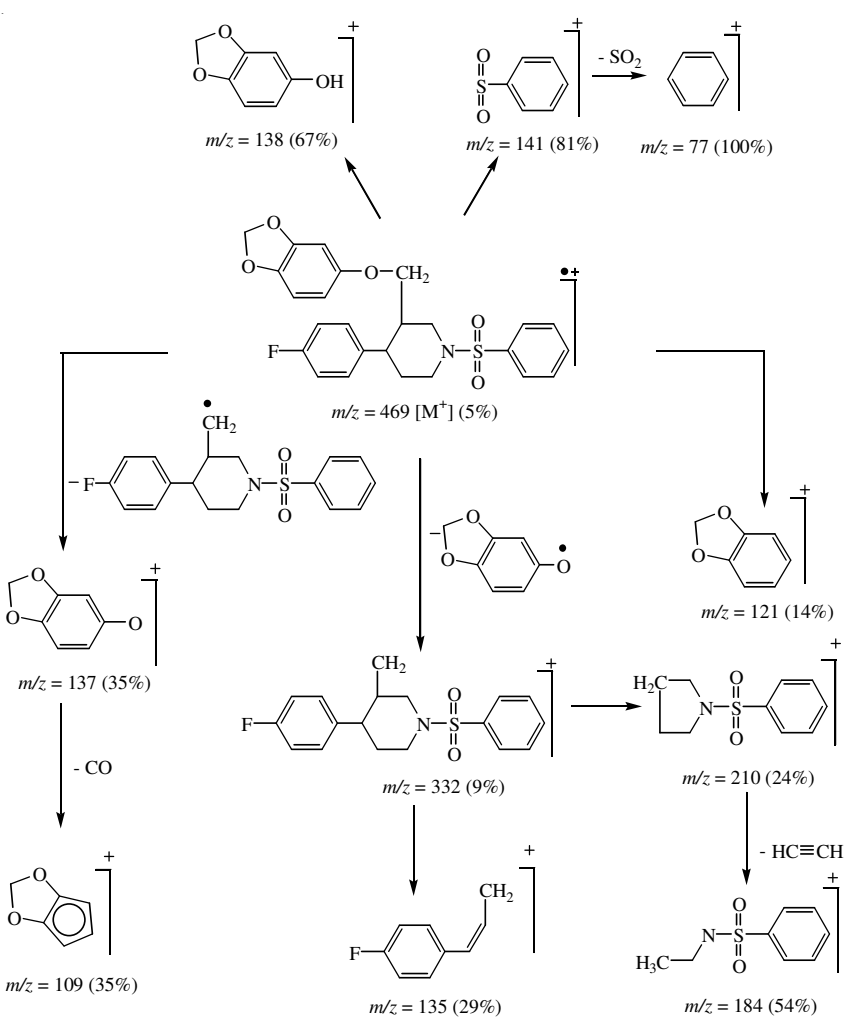

Fig. 1. Mass fragmentation pattern of $N$-(benzenesulfonyl)paroxetine (3a)

Enzyme inhibition activity: The screening of these synthesized compounds against lipoxygenase (LOX) enzymes revealed that these molecules exhibited moderate inhibitory potential as it was evident from their $\mathrm{IC}_{50}$ values. The results are given in Table-1. The most active inhibitor of lipoxygenase enzyme, $N$-(benzylsulfonyl)paroxetine (3I) showed $\mathrm{IC}_{50}$ value of $128.9 \pm$ $0.03 \mu$ moles/L relative to Baicalein, a reference standard having

\begin{tabular}{|c|c|c|c|}
\hline \multicolumn{4}{|c|}{$\begin{array}{l}\text { TABLE-1 } \\
\text { LIPOXYGENASE ACTIVITY OF THE } N \text {-ARALKYL/ } \\
\text { ARYL SULFONYL PAROXETINE 3a-n }\end{array}$} \\
\hline Compound No. & Conc. (mM) & Inhibition (\%) & $\mathrm{IC}_{50}(\mu \mathrm{M})$ \\
\hline $3 a$ & 0.5 & $16.57 \pm 0.61$ & - \\
\hline 3b & 0.5 & $93.98 \pm 0.05$ & $301.9 \pm 0.05$ \\
\hline $3 c$ & 0.5 & $59.92 \pm 0.03$ & $>500$ \\
\hline $3 d$ & 0.5 & $17.17 \pm 0.13$ & - \\
\hline $3 e$ & 0.5 & $58.86 \pm 0.04$ & $>500$ \\
\hline $3 f$ & 0.5 & $40.84 \pm 0.17$ & - \\
\hline $3 g$ & 0.5 & $34.78 \pm 0.07$ & - \\
\hline $3 h$ & 0.5 & $98.01 \pm 0.01$ & $171.9 \pm 0.07$ \\
\hline $3 \mathbf{i}$ & 0.5 & $21.01 \pm 0.15$ & - \\
\hline $3 \mathbf{j}$ & 0.5 & $61.23 \pm 0.05$ & $437.5 \pm 0.003$ \\
\hline $3 \mathbf{k}$ & 0.5 & $24.08 \pm 0.07$ & - \\
\hline 31 & 0.5 & $89.38 \pm 0.01$ & $128.9 \pm 0.03$ \\
\hline $3 m$ & 0.5 & $93.78 \pm 0.03$ & $181.8 \pm 0.007$ \\
\hline $3 \mathbf{n}$ & 0.5 & $63.69 \pm 0.01$ & $432.7 \pm 0.01$ \\
\hline Baicalein & 0.5 & $93.79 \pm 1.27$ & $22.41 \pm 1.3$ \\
\hline
\end{tabular}

Note: $\mathrm{IC}_{50}$ values (concentration at which there is $50 \%$ enzyme inhibition) of compounds were calculated using EZ-Fit Enzyme kinetics software (Perella Scientific Inc. Amherst, USA).
$\mathrm{IC}_{50}$ value of $22.4 \pm 1.3 \mu \mathrm{mol} / \mathrm{L}$. This inhibitory potential of $\mathbf{3 l}$ was due to the presence of aralkyl sulfonyl group. Out of these fourteen synthesized compounds, six remained inactive and two showed relatively very weak activity. The remaining active compounds can be given an order for their potential activity as, $\mathbf{3} \mathbf{l}>\mathbf{3} \mathbf{h}>\mathbf{3} \mathbf{m}>\mathbf{3} \mathbf{b}>\mathbf{3 n}>\mathbf{3} \mathbf{j}$. These compounds can further be exploited and their derivatives could be synthesized to acquire closer to $\mathrm{IC}_{50}$ values of the standard, baicalein. In this way, the compounds could be potential target in the drug invention and drug improvement program.

\section{Conclusion}

All of the compounds were synthesized in a facile and environmentally benign method. The projected structures of the compounds are well supported by spectroscopic data. From the enzyme inhibition data (Table-1), it might be concluded that the compounds have moderate activity against lipoxygenase enzyme as it was evident from their $\mathrm{IC}_{50}$ values, relative to the standard used.

\section{ACKNOWLEDGEMENTS}

The authors are thankful to Higher Education Commission of Pakistan for providing financial assistance.

\section{REFERENCES}

1. S.P. Runyon, J.P. Burgess, P. Abraham, K.I. Keverline-Frantz, J. FlippenAnderson, J. Deschamps, A.H. Lewin, H.A. Navarro, J.W. Boja, M.J. Kuhar and F.I. Carrol, Bioorg. Med. Chem., 13, 2439 (2005).

2. K. Sugi, N. Itaya, T. Katsura, M. Igi, S. Yamazaki, T. Ishibashi, T. Yamaoka, Y. Kawada, Y. Tagami, M. Otsuki and T. Ohshima, Chem. Pharm. Bull. 48, 529 (2000).

3. J. Cossy, O. Mirguet, D. Gomez Pardo and J.-R. Desmurs, Tetrahedron Lett., 42, 5705 (2001).

4. A. Alsughayer, A.A. Elassar, S. Mustafa and F. Sagheer, J. Biomater. Nanobiotechnol., 2, 144 (2011).

5. J.M. Baskin and Z. Wang, Tetrahedron Lett., 43, 8479 (2002).

6. S. Kumar, M.S. Niranjan, K.C. Chaluvaraju, C.M. Jamakhandi and D. Kadadevar, J. Curr. Pharm. Res., 1, 39 (2010).

7. N. Ozbek, H. Katircioglu, N. Karacan and T. Baykal, Bioorg. Med. Chem., 15, 5105 (2007).

8. F. Shi, M.K. Tse, S. Zhou, M.-M. Pohl, J. Radnik, S. Hübner, K. Jähnisch, A. Brückner and M. Beller, J. Am. Chem. Soc., 131, 1775 (2009).

9. M.A. Abbasi, V.U. Ahmad, M. Zubair, M.A. Rashid, U. Farooq, S.A. Nawaz, M.A. Lodhi, T. Makhmoor, M.I. Choudhary and Atta-urRahman, Proc. Pak. Acad. Sci., 42, 121 (2005).

10. G.A. Alitonou, F. Avlessi, D.K. Sohounhloue, H. Agnaniet, J.M. Bessiere and C. Menut, Int. J. Aromather., 16, 37 (2006).

11. R.S. Byrum, J.L. Goulet, R.J. Griffiths and B.H. Koller, J. Exp. Med., 185, 1065 (1997).

12. H. Khalid, Aziz-ur-Rehman, M.A. Abbasi and K.M. Khan, Int. J. Pharm. Pharm. Sci., 4, 443 (2012).

13. Aziz-ur-Rehman, S. Afroz, M.A. Abbasi, W. Tanveer, K.M. Khan, M. Asraf, I. Afzal and N. Ambreen, Pak. J. Pharm. Sci., 25, 809 (2012).

14. S. Baylac and P. Racine, Int. J. Aromatherap., 13, 138 (2003).

15. P. Jakubec, D.M. Cockfield, M. Helliwell, J. Raftery and D.J. Dixon, Beilstein J. Org. Chem., 8, 567 (2012). 